\title{
Las raíces cartesianas del naturalismo epifenomenalista: el caso de Thomas H. Huxley ${ }^{*}$
}

\author{
Cartesian Roots of Epiphenomenalist Naturalism: The Thomas H. Huxley Case
}

Ricardo Mejía Fernández ${ }^{\dagger}$

\begin{abstract}
Resumen
El autor nos presenta la recepción cartesiana del británico Thomas H. Huxley (1825-1895), uno de los científicos evolucionistas más influyentes de finales del siglo XIX y principios del XX, y que ha tenido grandes repercusiones en la filosofía de la ciencia y de la mente. El resultado de este artículo es mostrar la defensa filosófica por parte de Huxley de un naturalismo epifenomenalista, que llega hasta nuestros días y que debe mucho al trabajo de René Descartes en lo que concierne a la constitución mecánica y autónoma de la naturaleza, pero que se aleja de él al concebir al cerebro como órgano de la mente y a la consciencia como producto colateral suyo.
\end{abstract}

Palabras clave: Huxley - Descartes - naturalismo - epifenomenalismo

\begin{abstract}
The author shows the Cartesian reception made by Thomas H. Huxley (1825-1895), one of the most influential evolutionary scientists of the late nineteenth and early twentieth century, which has had an enormous impact on the philosophy of science and mind. The result of this article is giving an account of the Huxley's philosophical defense of an epiphenomenalist naturalism, which comes to the present day and which owes too much to the work of René Descartes in relation to the mechanical and autonomous constitution of Nature. Huxley, on the contrary, moves away from him in conceiving the brain as the organ of mind and in understanding consciousness as its collateral product.
\end{abstract}

Keywords: Huxley - Descartes - naturalism - epiphenomenalism

\footnotetext{
* Recibido: 2 de Enero de 2014. Aceptado en versión revisada: 28 de Marzo de 2014.

† Universidad de Salamanca, España. Para contactar al autor, por favor, escriba a: ricardomejia@usal.es. Metatheoria 4(2)(2014): 61-80. ISSN 1853-2322.

(C) Editorial de la Universidad Nacional de Tres de Febrero. Publicado en la República Argentina.
} 


\section{Introducción}

En el presente artículo nos centraremos en sacar a la luz las raíces cartesianas del naturalismo fenomenalista a partir de la obra del científico británico Thomas Henry Huxley (1825-1895). En otras palabras, será nuestra misión investigar la recepción que Huxley hizo de la filosofía del francés René Descartes (1596-1650), manteniéndonos en la originalidad del pensamiento huxleyano.

Este artículo quiere ser novedoso en dos campos: la historia de la ciencia y la filosofía de la mente. En lo primero, queremos estudiar y dar a conocer los altos conocimientos filosóficos, en especial de la filosofía cartesiana, de uno de los científicos evolucionistas más importantes de la historia. En lo segundo, queremos presentar la teoría de la consciencia colateral dentro del más reciente debate epifenomenalista. Como veremos en las páginas que siguen, Huxley se decantó -y fue uno de sus más potentes impulsores- por un naturalismo que constituye el terreno general en el que edifica su manera de pensar y que, en lo que atañe a la consciencia, se caracteriza por un acentuada deriva epifenomenalista.

De este modo, dividiremos nuestro artículo en dos secciones con una temática propia. Así pues, la primera (1) estará dedicada al proyecto naturalista global de Huxley. No en vano diremos que es en él en donde se enmarca su investigación de la mente consciente. Este primer punto descubriremos las principales líneas filosóficas naturalistas que Huxley hereda de Descartes (1.1), viendo cómo el francés fue uno de los más influyentes naturalistas de la modernidad. Intentaremos clasificar a Huxley, en la medida en que nos lo permita el científico británico, en algunas de las variantes contemporáneas del naturalismo.

En la segunda parte (2) pondremos nuestra atención en la consciencia en cuanto que epifenómeno de la naturaleza, destacando los puntos en los que el británico discrepó del pensador francés y en los que, digámoslo así, también le supone una rica aportación. La teoría del automatismo consciente (2.1.) le servirá a Huxley para criticar la teoría cartesiana a favor de unos animales desprovistos de actos conscientes y establecer, así, un único tipo de causalidad eficiente para dichos actos. En la tercera parte (3) acabaremos, finalmente, con unas sucintas conclusiones, en la que destacaremos a un Huxley temeroso de identificar los actos mentales con los actos físicos pero aun así empoderado para concluir la impotencia causal de unos sobre los otros.

\section{El proyecto naturalista de Huxley}

El naturalismo -diríamos desde una perspectiva general- es la actividad teórica y/o práctica que consiste en hacer que el terreno de todas, o en algunos autores de casi todas, las dimensiones de la vida humana sea la naturaleza, tal y como es aprehendida por las ciencias experimentales. Mantiene, pues, muy vivo parte del enfoque epistemológico empirista, como nos los confirmaba Quine al apuntar que "the most notable norm of naturalized epistemology actually coincides with that of traditional epistemology. It is simply the watchword of empiricism: nihil in mente quod non prius in sensu" (Quine 1992, p. 19).

El naturalismo, no sólo en las teorías del conocimiento, ha sido y es objeto de un amplio debate. Antes de zambullirnos en la lectura de Huxley, dediquemos a esto unas breves líneas introductorias. No existe un definición unívoca de "naturalismo", por lo que, como veremos en nuestro científico, la idea que obtengamos del naturalismo se desprenderá de multitud de afirmaciones y planteamientos desperdigados en nuestro autor. Generalmente -y aquí nos servimos de la investigación de Papineau (2007) - nos encontramos con tres grandes formas de naturalismo, las cuales dan lugar a otras subformas o se entremezclan con ellas:

1) El naturalismo general. Es el que apuntábamos al principio y fue adoptado en la primera mitad del siglo XX por Dewey, Nagel, Hook o Sellars, los cuales "urged that reality is 
exhausted by nature, containing nothing 'supernatural', and the scientific method should be used to investigate all areas of reality, including 'human spirit'" (Papineau 2007). En nuestro artículo veremos cómo Huxley, ya en el siglo XIX y como predecesor indiscutible, fue poniendo los fundamentos del candoroso debate contemporáneo sobre este tipo de naturalismo.

2) El naturalismo ontológico. Es la especificación "fuerte" (strong) del naturalismo general. Según estos naturalistas, lo que ha de guiar cualquier investigación son "the contents of reality, asserting that reality has no place for 'supernatural' or other 'spooky' kinds of entity" (Papineau 2007). Una versión muy característica es el naturalismo "fisicalista" (Churchland o Davidson son exponentes, como veremos más adelante), según la cual los estados mentales son idénticos a los estados físicos del cerebro. Se trata de una versión reductiva pues los fisicalistas aseguran tener no sólo tener certeza sino evidencia de que tanto la mente como sus operaciones son exclusivamente físicas.

3) El naturalismo metodológico. Se trata de lo que recientemente se viene llamando "un naturalismo evolutivo modesto", como el de Roland N. Giere. ${ }^{1}$ Aquí nos encontramos, más bien, con un "compromiso" en el método científico cuyo prisma es el de las ciencias experimentales, aceptando su provisionalidad y demostrando reservas hacia hacer afirmaciones determinantes sobre el ser de las cosas.

Huxley fue un naturalista convencido, tanto de vocación como de profesión. En su famosa y polémica obra El puesto del hombre en la naturaleza, escrita en $1863,{ }^{2}$ el británico habla de una naturaleza en mayúscula, no sólo por el uso de esta forma de escritura, sino por la importancia que le concede en la ciencia y en la teoría de la evolución de las especies, en especial de la humana. Yendo mucho más allá que Darwin, nuestro autor polemizó fuertemente con los antievolucionistas y fue quien popularizó la idea de nuestra descendencia ancestral a partir de los simios. Leemos en el británico:

As if to demonstrate, by a striking example, the impossibility of erecting any cerebral barrier between man and the apes, Nature has provided us, in the latter animals, with an almost complete series of gradations from brains little higher than that of a Rodent, to brains little lower than that of a man (Huxley 2006, p. 64).

La Naturaleza es la que nos provee, en los homínidos, de las progresivas transformaciones orgánicas, en este caso, cerebrales, que es identificable desde el enfoque evolucionista. Es decir, si ella es el terreno fundamental que nos une a los seres inferiores, entonces es imposible que haya insalvables barreras entre ellos y nosotros. La Naturaleza es, pues, una y la misma para todos, es proveedora y nos preside siempre. Ella es, en definitiva, creadora, como lo escribía Huxley:

Nature, by this process, has attained much the same result as that at which a human artificer arrives by his operations in a brick field. She takes the rough plastic material of the yelk and brakes it upon into well-shaped tolerably even-sized masses - handy for building up into any part of the living edifice (Huxley 2006, p. 40).

El británico compara a la Naturaleza con un artífice, que nos recuerda al relato creacionista del Génesis bíblico en el que el Creador aparece como un alfarero que modela de los elementos terrenos a los seres vivos. A partir de la yema del huevo celular (el núcleo, en términos técnicos), ella es capaz de construir, mediante todo un conjunto de divisiones y subdivisiones, todo el edificio de la vida en la diversificación más fascinante de sus seres. Pero estas comparaciones son solamente imágenes.

Nuestro autor abogará por la teoría evolucionista darwiniana pues ve en la misma sendos fundamentos científicos. Huxley cree en la ciencia y en su método experimental: no puede ocultar su fascinación por una ciencia que, ya en el siglo XIX, empieza a ser protagonista de grandes

\footnotetext{
1 "For any aspect of the world, seek a naturalistic rather than a super naturalistic explanation. It is a virtue of a methodological stance that its adoption does not even seem to require an a priori justification. Commitment to the method can be somewhat justified by appeal to past successes at finding naturalistic explanations" (Giere 2007, p. 23).

2 Man's Place in Nature. The Evolution Debate: 1813-1870, edición citada Huxley (2006).
} 
descubrimientos. Es, pues, una postura típicamente naturalista concebir a la ciencia empírica como la principal reveladora de verdad:

\begin{abstract}
Science has fulfilled her function when she has ascertained and enunciated truth; and were these pages addressed to men of science only, I should now close this essay, knowing that my colleagues have learned to respect nothing but evidence, and to believe that their highest duty lies in submitting to it, however it may jar against their inclinations (Huxley 2006, p. 73).
\end{abstract}

La ciencia se mueve por evidencias y Huxley sabe bien que su trabajo está dirigido a aquellos que no sólo la tienen en estima sino que obedecen sus dictámenes universales. La mayor tarea de los científicos es, según el británico, someterse a la evidencia que nace de una ciencia que trabaja con los datos provenidos del mundo. Esta actitud, aunque parezca dogmática, no es más que reconocer lo que se da; lo cual acabará, algún día, por aportarnos lo más grande. Huxley, a diferencia de las opciones científicas del naturalismo ontológico fuerte, reconoce que la ciencia no posee en plenitud la verdad, sino que se aproxima a ella:

Time proves each reply to have been a mere approximation to the truth-tolerable chiefly on account of the ignorance of those by whom it was accepted, and wholly intolerable when tested by the larger knowledge of their successors (Huxley 2006, p. 37).

Para completar el presente planteamiento, no podemos dejar de referirnos a una fuente posterior y no menos importante del pensamiento huxleyano: nos referimos a sus Sermones laicos, que fueron publicados en 1870. ${ }^{3}$ En ellos, leemos que "the ultimate court of appeal is observation and experiment, and not authority [...] teaching it to estimate the value of evidence" (Huxley 2009a, p. 130). A liberarnos de las cadenas de la autoridad, la Naturaleza es el más prestigioso ateneo a la que puede aspirar ser alumno cualquiera que esté en sincera búsqueda de la verdad. El mundo conserva su frescura sempiterna y puede ofrecer experimentalmente continuas novedades a la mirada atenta de cualquier ser humano, no sólo del investigador, si bien este último estará en condiciones de hacer ciencia en cuanto tal.

Así lo afirmaba Huxley en un bello texto, sosteniendo que el estudio de la naturaleza (¡y de ser educado por ella!) es fuente de felicidad:

Nature is still continuing her patient education of us in that great university, the universe, of which we are all members [...]. Those who take honours in Nature's university, who learn the laws which govern men and things and obey them, are the really great and successful men in this world. (Huxley 2009a, p. 38)

Nuestro autor, en definitiva, es un partidario explícito de un naturalismo obediencial, más como método que como una afirmación claramente metafísica. En 1892, el británico redactaba, en el prólogo a sus Ensayos sobre algunas cuestiones controvertidas, ${ }^{4}$ que toda la historia del pensamiento había transcurrido como una encarnizada batalla entre la corriente naturalista y la -así denominada por élsupernaturalista. Pero esto no consiste en unas afirmaciones de corte maniqueo: no se trata de una simple oposición. En referencia a los albores del siglo XVIII, Huxley se queja del hundimiento del naturalismo por una corriente que se jactaba de haber hallado la verdad en lo que está allende el universo:

Thus, by degrees, the freethinking, or the indifference, prevalent among us in the first half of the eighteenth century, was replaced by a strong supernaturalistic reaction, which submerged the work of the free-thinkers; and even seemed for a time, to have arrested the naturalistic movement of which that work was an imperfect indication (Huxley 2009b, p. 18).

El libre-pensamiento se ha visto, por tanto, dañado por el supernaturalismo, el cual si bien presume de perfección, carece de ella. El naturalismo fue, como lo cree nuestro autor, introducido por los arios

\footnotetext{
3 Lay Sermons, Adresses and Reviews, edición citada Huxley (2009a).

${ }^{4}$ Essays upon some Controversed Questions, edición citada Huxley (2009b).
} 
jónicos, es decir, por aquellos filósofos que identificaban el arjé cósmico con un elemento material concreto. ${ }^{5}$ Lo cierto es que el oscilar histórico entre el naturalismo y ese movimiento "is an indication of the progress, or of the regress, of humanity; of a fall from, or an advance towards, the higher life" (Huxley 2009b, p. 6). El ideal del progreso está muy arraigado en un Huxley que comparaba la actitud no indulgente con la Naturaleza con una caída, como si se tratase de un pecado original que hubiese desencadenado para la humanidad todo tipo de desastres y calamidades.

¿Pero qué es lo que nuestro autor niega del supernaturalismo? Lo que Huxley no puede tolerar no es que existan estos o aquellos entes sobrenaturales: él no es propiamente un naturalista ontológico. Sobre esta cuestión no se posiciona de manera directa. Nuestro autor se opone, por el contrario, a la demanda supernaturalista (en alusión sobre todo a la denostada teología natural) de que sus argumentos posean el mismo nivel de evidencia que el que nos proporciona la ciencia natural (Huxley 2009b, p. 35). Este rasgo sólo lo encontramos en la Naturaleza porque puede ser captada por los sentidos. La Naturaleza, pues, "covers the totality of what it is" (Huxley 2009b, p. 35).

Así pues, el naturalismo huxleyano, como todo naturalismo que se precie, tiene una pretensión de totalidad; si bien esta pretensión es enlazada por nuestro autor, modestamente y en la medida en que la ciencia progresa, con el reino experiencial. Es un naturalista metodológico. Pero también se aprecia un cierto compromiso óntico, en la región de lo que es, al menos en el ámbito de lo fáctico, sin posicionarse acerca de lo ultraterreno. No olvidemos que dicho compromiso entronca con su metodología más que con las consecuencias metafísicas del naturalismo ontológico.

A veces se ha acusado a Huxley de haber infectado el campo de la ética de la denominada falacia naturalista ${ }^{6}$ divulgada por George E. Moore en sus Principia Ethica (Moore 1913), a partir de su análisis de la obra de David Hume. ${ }^{7}$ De una manera bastante precipitada e informal, la falacia naturalista es "generalmente y de forma muy sintética, la posibilidad de deducir enunciados éticos, proposiciones normativas de la vida práctica, a partir de la realidad" (Rus 1994, p. 322). Así las cosas, se quiere fundamentar el deber moral a partir del ser, ora en términos naturales, ora en sobrenaturales. ¿Incurrió en esto Huxley?

Encontramos en Huxley textos en los que afirma cierta moralidad en la naturaleza que requiere "a training in the theory and practice of obedience to the moral laws of Nature" (Huxley 2009a, p. 41). Aún más contundente se muestra nuestro autor cuando ensalza la Revolución Científica decimonónica porque "is creating a firm and living faith in the existence of immutable moral and physical laws, perfect obedience to which is the highest possible aim of an intelligent being" (Huxley 2009a, p. 130). Mediante estas candentes citas, nadie dudaría que el británico aboga por conectar lo moral y lo natural, mas no podemos precipitarnos: el tipo de leyes se tienen que concretar, no siendo suficiente hace afirmaciones, sin más.

Hemos visto que, en Huxley, de nuestra relación con la Naturaleza depende que lleguemos o no a la felicidad, la cual ha sido entendida como el bien más grande de cada uno. Nuestro autor, de igual forma, encontró en las ideas de Darwin una gran fuente de inspiración. Por ello, no somos quienes para descartar que la teoría de la evolución darwiniana haya tenido consecuencias en el campo moral, en especial en lo concerniente a la conducta de los grupos humanos. Huxley dio cuenta del surgimiento de instintos altruistas, mediante un proceso en el que el grupo ejercía una tarea selectiva, que no es otra cosa que la selección natural operando en el nivel comunitario, y no tanto en el individual (White 2003, p. 168). Pero esto es ciencia no una ética filosófica.

\footnotetext{
5 "[S]cientific naturalism took its rise among the Aryans of Ionia. It would be difficult to find another three centuries which have given birth to four events of equal importance" (Huxley 2009c, p. 49).

${ }^{6}$ Paul White nos los confirma: "Huxley has often been viewed as an early exponent of the 'naturalistic fallacy', the principle that it is illegitimate to derive moral principles from matters of fact" (White 2003, p. 51).

${ }^{7}$ Una definición muy general (que versa sobre la ética de David Hume pero que nos vale como noción básica) fue la que proporcionó J. Mackie: "Writers on morality, Hume noted. often move imperceptibly from statements joined by 'is' to ones joined by 'ought' and 'ought not'. These, he protests, express some new relation, make some new sort of claim. Which needs to be explained: 'should be given, for what seems altogether inconceivable, how this new relation can be a deduction from others which are entirely different from it'" (Mackie 1990, p. 64).
} 
Por ello y para concluir este apartado, estamos obligados a reconocer que Huxley "saw no way to explain, by the same evolutionary mechanism, the conversion of these instincts into ethical norms, applied universally to humankind" (White 2003, p. 168). Tuvo, finalmente, el británico intuiciones ontológicas que jamás desplegó y engarzó racionalmente, al modo kantiano, en una moral universal. Que tal vez Huxley pudo haberlo hecho porque se seguía de su pensamiento es un asunto que, al estar en la esfera de lo hipotético, excede nuestra investigación. Lo que sí podemos establecer es que el británico mantuvo, sobre todo, un proyecto naturalista general y metodológico.

\subsection{La herencia naturalista cartesiana}

Huxley demostró un gran reconocimiento hacia el pensador galo, situándolo en un lugar central de la historia de la filosofía y también de la ciencia. Así lo escribía en una carta dirigida en 1870 a la Sociedad Cristiana de Hombres Jóvenes de Cambridge, la cual, más tarde, será publicada en el McMillan's Magazine: "It seems to me that the thinker who, more than any other, stands in the relation of such a stem towards the philosophy and the science of the modern world is René Descartes" (Huxley 2009a, p. 352).

Huxley considera que el espíritu de Descartes está presente por doquier en el pensamiento, no únicamente de sus contemporáneos franceses, sino marcando decididamente la reflexión futura. Nuestro autor no duda en llamarlo sin duplicidades "the Philosopher" (Huxley 2009a, p. 352). El mayor mérito que el británico atribuye a Descartes, refiriéndose en este caso al Discurso del método (Descartes 1637), no es la introducción del tan divulgado racionalismo, sino que se trata, curiosamente, el de haberse opuesto a la metafísica más recalcitrante, recuperando los fundamentos materiales de las ideas:

[T]he 'Discourse' shows us another, and apparently very different, path which leads, quite as definitely, to that correlation of all the phenomena of the universe with matter and motion, which lies at the heart of modern physical thought, and which most people call Materialism (Huxley 2009a, p. 362).

Huxley se aleja de la interpretación cartesiana canónica de la idea desencarnada para adentrarse en el Descartes físico (o, en su tiempo, denominado "mecánico"), es decir, para retrotraernos al desconocido introductor de una inaudita filosofía de la materia, matriz del naturalismo moderno, como mínimo en su versión metodológica. El galo tuvo la habilidad de mostrar, a partir de los descubrimientos de Galileo, que las porciones más remotas del universo "were governed by mechanical laws" (Huxley 2009a, p. 363). Sin embargo, conviene tener en claro que el materialismo, que en lo sucesivo adoptará Huxley, constituye más bien un método en lo que conforma su filosofía de la naturaleza. Según nuestro autor, Descartes adelantó severos conocimientos de la fisiología moderna, en lo que respecta a la consideración de las mociones corporales como solos fenómenos físicos.

Todas las funciones que comparten tanto los animales como los hombres están ejecutadas por el cuerpo como un mero mecanismo. En el próximo apartado veremos en qué afecta esto a la mente, punto en el que nuestro científico se distanciará de Descartes. Por ahora nos es suficiente con enunciar, con Huxley, que encontramos en el francés más universal, debido a su concepto de res extensa, un antecedente claro del naturalismo y, aún más, del materialismo:

In truth, Descartes' physiology, like the modern physiology of which it anticipates the spirit, leads straight to Materialism, so far as that title is rightly applicable to the doctrine that we have no knowledge of any thinking substance, apart from extended substance (Huxley 2009a, pp. 370-371).

¿Y qué nos dice el propio Descartes? Huxley no iba nada desencaminado pues esta cuestión la encontramos tanto en el Corpus mayor como en la correspondencia privada cartesiana, en la cual tiene oportunidad de aclarar los aspectos más complejos de su pensamiento. En una carta de marzo de 1638 y en un exacerbado enfoque dualista, el pensador escribe en un barroco francés:

De cela seul qu'on conçoit clairement \& distinctement les deux natures de l'ame \& du corps comme diuerses, on connoist que véritablement elles sont diuerses, et par consequent que l'ame peut penser 
sans le corps, nonobstant que, lors qu'elle lui est jointe, elle puisse estre troublée en ses opérations par la mauuaise disposition des organes (Descartes 1638, p. 38). ${ }^{8}$

Que el alma y el cuerpo son dos naturalezas diferentes es algo bien comentado y conocido en Descartes y, de un modo enigmático, el que estuviesen unidos por la glándula pineal. Movido por su racionalismo, el galo concibe que el alma pueda pensar sin la ayuda corporal, si bien no puede evitar afirmar que el cuerpo pueda dar problemas al pensamiento, según sea el funcionamiento de sus órganos. Esto es debido a que el francés se inscribe en una tradición que entiende el cuerpo -y lo material, al fin- como una carga: en las Meditationes de Prima Philosophia (Descartes 1641), ${ }^{9}$ Descartes ve a la naturaleza como fuente de confusión y, lo que es peor, como un alter con respecto a su ser pensante. Confesaba el galo en su obra mayor: "Cum enim viderer ad multa impelli a natura, quae ratio dissuadebat, non multum fidendum esse putabam iis quae a natura docentur” (Descartes 2009, p. 174). ${ }^{10}$

Lo más interesante es que Descartes, queriendo salvar la autonomía del espíritu, relega indirectamente el mundo natural a un ámbito también diferente y, en la medida de lo posible, autónomo. Se trata de la res extensa, como comprobamos en sus póstumas Dos opúsculos. Reglas para la dirección del espiritu. Investigación de la verdad: "entendemos por extensión todo aquello que tiene longitud, latitud y profundidad” (Descartes 1959, p. 173). Se trata de una concepción de la naturaleza física como aquello que es susceptible de ser medido y de ocupar espacio. Descartes irá succionando del mundo natural todo valor espiritual de suyo, desecando -valgan las metáforas- la savia ontológica que la metafísica clásica le había inyectado. Y aún nos quedamos cortos en sacar a relucir cómo al galo se le pasa por la cabeza que esta extensión fuese, por simple hipótesis, algo ajeno a la idea: "Porque aunque alguno pueda persuadirse, por ejemplo, que, aún en el caso de que sea reducido a la nada todo lo que es extenso en la naturaleza, no repugna a pesar de ello que la extensión misma exista por sí sola" (Descartes 1959, p. 173).

Aquí empieza el naturalismo. Nos dice en el Discurso del método: "[I]'ay remarqué certaines loix, que Dieu a tellement éstablies en la nature" (Descartes 1637, p. 41), ${ }^{11}$ acto que ha sido como una impresión que dota a lo impreso de una autonomía. Es la questio disputata medieval en torno a si Dios se limita a crear o también conserva las cosas en el ser. La conservación es una doctrina que el francés adopta para no mostrar en lo que en la contemporaneidad se ha llamado pandeísmo, es decir, un Dios que, en el acto creacional, abdica de su ser en lo creado. Sin la doctrina conservacionista, pues, podríamos llegar a creer que la Naturaleza se ha autogenerado, por lo que Descartes nos señala que el ente divino:

[P] ouruû qu'ayant establi les Loix de la Nature, luy prestast son concours, pour agir ainsi qu'elle a de coustume, on peut croyre, sans faire tort au miracle de la création, que par cela seul toutes les choses qui sont purement matérielles auroient pu, avec le temps, s'y rendre telles que nous les voyons à present (Descartes 1637, p. 45). ${ }^{12}$

Mas al galo se le pasa por alto dar cuenta racional de esta conservación y, cada vez más, se aproxima a la tesis de que hay cosas puramente materiales, si bien la fuerza que las mueve se debe, en último término, a un Dios que, al comienzo de los tiempos, ha impreso al reino natural de unas leyes para su desarrollo. La duda adviene a nuestra mente cuando nos preguntamos qué pasa una vez que esta suerte de desarrollo llega al estadio actual. ¿No será un recurso de Descartes para ahuyentar las sospechas de

8 "De ahí sólo que concibamos claramente y distintamente las dos naturalezas del alma y del cuerpo como diversas, conocemos que verdaderamente son diversas, y por consiguiente, que el alma puede pensar sin el cuerpo, pese a que, mientras le está unida, puede ser enturbiada en sus operaciones por la mala disposición de los órganos”.

9 Meditaciones acerca de la filosofía primera. Seguidas de objeciones y respuestas, edición del 2009.

10 "Porque como me veía impulsado por la naturaleza a muchas cosas de las que disuadía la razón, no creía que hubiera que confiar mucho en las cosas que son enseñadas por la naturaleza" (Descartes 2009, p. 175).

11 "[Y] he remarcado ciertas leyes que Dios ha verdaderamente establecido en la naturaleza".

12 "[C]on tal que, habiendo establecido las Leyes de la Naturaleza, le prestase su concurso, para actuar de la forma acostumbrada, podríamos creer, sin menoscabar el milagro de la creación, que por esto todas las que son puramente materiales hubiesen podido, con el tiempo, volverse tal y como las vemos en el presente". 
herejía de los teólogos de la Sorbona o, quizá, una necesidad imperiosa de mantener un dogma de quien fue un piadoso católico? Si, por un lado, Descartes insinúa este concurso divino (¿no adolece de un pronunciado extrincesismo?), manifiesta que la Naturaleza cuenta, ya y ahora, con las leyes necesarias para ser ella en puridad. El mismo Huxley se percatará de las sospechas de ateísmo en torno al galo pues "his old friends the Jesuits put his works upon the "Index' ${ }^{13}$ and called him an Atheist; while the Protestant divines of Holland declared him to be both a Jesuit and an Atheist" (Huxley 2009a, p. 375).

Pero nos vemos impedidos a disimular nuestra perplejidad al notar que el galo se mostrase, en aquella época pre-darwiniana, partidario de una naturaleza en constante transformación; si bien no conocía ni imaginaba la posterior teoría de la evolución. Huxley, aún admirando este último punto en el filósofo francés, consideró esta postura como demasiado precoz. Fue, en efecto, admirable "the attempt of Descartes to get at a theory of the universe by the same a priori road; but, in my judgment, they are as premature" (Huxley 2009b, p. 38). Situar la evolución de las especies como un punto de partida a priori no es una actitud propiamente científica. Al menos -y esto es lo que resalta Huxley-, Descartes, adelanta muchas de las tesis decimonónicas, por lo que nos dice que prefiere una Naturaleza cambiante a una que permanezca estática, en flagrante oposición a la cosmología aristotélica: "Et leur nature est bien plus aysée à conceuoir, lorsqu'on les voit naistre peu à peu en cette sorte, que lorsqu'on ne les considere que toutes faites" (Descartes 1637, p. 45). ${ }^{14}$

Así pues, en otra misiva sobre los animales y pese a que reconoce la insuficiencia de su ser respecto al ente divino, escribe que "cette Nature agit en tout suiuant les loix exactes des Mechaniques" (Descartes 1639, p. 535). ${ }^{15}$ Los animales son análogos a los entes regidos por principios mecánicos exactos, estando desprovistos de alma. En la carta citada anteriormente, el filósofo galo había concedido, en el lenguaje no formal y no oficial que supone una carta, atributos divinos a la Naturaleza, personificándola de igual modo como hemos podido apreciar en Huxley:

[E]lle les remplit [a las plantas] d'une infinité de petits conduits imperceptibles à la veuë, par lesquels elle fait monter peu à peu certaines liqueurs, qui, estant paruenuës au haut de leurs branches, s'y mêlent, s'y agencent, \& s'y desseichent en telle façon qu'elles y forment des feuilles, des fleurs \& des fruits (Descartes 1638, p. 40). ${ }^{16}$

A causa de los textos que hemos traído a colación, Huxley no vaciló ni un segundo en otorgarle a Descartes la patria potestad del naturalismo, el cual tanto auge tomará en los en los tiempos venideros, viendo en su persona la primera en acometer una elaboración filosófica de este movimiento, cada vez más influyente en el siglo XIX:

It is important to note that the principle of the scientific Naturalism of the latter half of the nineteenth century, in which the intellectual movement of the Renascence has culminated, and which was first clearly formulated by Descartes (Huxley 2009b, p. 35).

\section{El epíteto "epifenomenalista" o en qué Huxley discrepó de Descartes}

\subsection{El epifenomenalismo}

En filosofía de la mente, el epifenomenalismo se relaciona con los estados mentales desde el punto de vista de su causación. Esta corriente no sólo se pregunta cuál es el tipo de causación de los fenómenos sino que defiende que éstos se deben eventos neurofisiológicos, los cuales, una vez originados, no causan ni afectan por sí mismos al ámbito cerebral de donde nacen ni a ningún otro componente

${ }^{13}$ El Index librorum prohibitorum (o Índice de libros prohibidos) fue un catálogo publicado por la Iglesia Católica en el que constaban las obras consideradas de ortodoxia dudosa y de perniciosas consecuencias para la moral cristiana. Fue suprimido en 1966 por el papa Pablo VI.

14 "Y su naturaleza es mucho más fácil de concebir, cuando las vemos nacer poco a poco en esta suerte, que cuando las consideramos como todas hechas".

15 "Esta Naturaleza actúa en todo siguiendo las leyes exactas de los Mecánicos".

16 "[E]lla las llena [a las plantas] de una infinidad de pequeños conductos imperceptibles a la vista, por los cuales ella hace subir poco a poco ciertos licores, que, habiendo llegado a lo alto de las ramas, allí se mezclan, se arreglan y se endurecen; de tal manera que forman hojas, flores y frutos". 
(Caston 1997). Si, por un lado, hallamos una causalidad antecedente, no hay ninguna casualidad física consecuente por parte de los estados mentales (epifenómenos). Expliquémonos mejor:

1. Todos los estados mentales están causados por eventos neurofisiológicos.

2. Una vez causados, adquieren un carácter cualitativamente distinto ${ }^{17}$ al neurofisiológico.

3. Luego, al no poseer la misma entidad causal, no pueden afectar a nada físico ni mucho menos revertir el proceso epifenomenal.

Si ejemplificamos esto, diríamos que si un sujeto cualquiera estuviese expuesto a las ondas musicales de una obra musical, las ondas sonoras, que atraviesan con sus vibraciones el conducto auditivo, estimularían las fibras del nervio estíbulo-coclear, cuyas células receptoras, localizadas en la membrana basilar del órgano de Corti, transformarían sinápticamente los datos sensoriales, llegando a la percepción del yo consciente, en cuanto experiencia cualitativa unificada, de las notas y compases rítmicos musicales. Este epifenómeno, incluso en los casos de experiencia estética, es de naturaleza distinta a las ondas sonoras y a la sinapsis neuronal, no pudiendo modificar ni lo anterior ni lo posterior a sí. En el caso de que la experiencia estética de la obra fuese negativa no contaríamos con la prerrogativa de la reversibilidad del estado mental negativo hacia los impulsos nerviosos neuronales antecedentes, ni mucho menos a las ondas musicales. No estaríamos -cáptese la ironía- en condiciones de recomponer dichos estímulos físicos para que así nos permitiesen mejorar materialmente la obra y ¿por qué no? nuestra percepción de la misma. Según el epifenomenalismo más tradicional, los estados mentales de fruición estética, aburrimiento, rechazo, etc., son "epifenómenos", es decir, productos venidos de lo neurofisiológico pero cualitativamente distintos, irreductibles e ineficaces.

En el siglo XIX, la teoría epifenomenalista tradicional fue introducida por Shadworth Hodgson en The Theory of Practice (1870), cuatro años antes que el importante artículo de Huxley, el cual más tarde comentaremos. El mismo término "epifenómeno" -inexistente en Huxley y del que se derivará "epifenomenalismo"- fue introducido, más tarde y aún en vida de nuestro autor, por el estadounidense William James en el primer volumen de sus Principios de psicología (James 1890), dándole un sentido específico, dentro de un monismo neutral hacia cualquier tipo de qualia mental que le llevó a fustigar a un Hodgson contra la función mecánica de la consciencia. ${ }^{18}$ James estaba en desacuerdo con él en que la consciencia fuese, al distinguirse del cerebro, comparable a algo inerte (inert), espumoso (foam) o a una áurea (aura). De todas maneras, este autor daba la razón a Huxley en ver en Descartes el precursor del naturalismo, en este caso de un naturalismo aplicado al carácter autosuficiente del sistema nervioso:

To Descartes belongs the credit of having first been bold enough to conceive of a completely selfsufficing nervous mechanism which should be able to perform complicated and apparently intelligent acts (James 2007, p. 130).

Sin embargo, el desacuerdo estriba en que los actos inteligentes fuesen aparentes y contrarios al principio de autosuficiencia biológica. El estadounidense opta por cargar en los hombros del galo el yugo de la arbitrariedad, sobre todo cuando trató a los animales de meros seres inanimados, así como al establecer que los actos más primarios del hombre estaban igualmente sine anima:

By a singularly arbitrary restriction, however, Descartes stopped short at man, and while contending that in beasts the nervous machinery was all, he held that the higher acts of man were the result of the agency of his rational soul (James 2007, p. 130).

17 "Distinto" lo oponemos conceptualmente a "diferente": la distinción significa, como en Descartes, lo advertido o distinguido en algo, mientras que lo diferente pertenece a otra res que es individualizada.

${ }^{18}$ Nos dice James refiriéndose a Hodgson: "But what on this view could be the function of the consciousness itself? Mechanical function it would have none. The sense-organs would awaken the brain-cells; these would awaken each other in rational and orderly sequence, until the time for action came; and then the last brain-vibration would discharge downward into the motor tracts. But this would be a quite autonomous chain of occurrences, and whatever mind went with it would be there only as an 'epiphenomenon' [las cursivas son nuestras], an inert spectator, a sort of 'foam, aura, or melody' as Mr. Hodgson says, whose opposition or whose furtherance would be alike powerless over the occurrences themselves" (James 2007, p. 129). 
Pero el problema en torno a si los animales eran puros seres mecánicos no fue una novedad de James, el cual no quiso aceptar el epifenomenalismo debido a que concebía toda la realidad como solamente natural. El monismo neutral, defendido por James en 1904 en iExiste la consciencia? sostiene que las propiedades de las cosas que conforman el mundo físico no tienen existencia propia en la consciencia que las experimenta sino en ellas mismas, sin gozar la mente de substancialidad distinta a la psique. Huxley, al contrario que el estadounidense, defendió que la consciencia es un algo emergente con características experienciales surgidas del cuerpo sentiente pero ya no modificadoras de nada corporal.

En El progreso de la ciencia, Huxley (1887) comparaba a los seres vivientes con una máquina de vapor que transforma en movimiento la energía que le es proporcionada, es decir, como un mecanismo cuya energía es transformada por el cerebro en actos cognitivos de cualidad distinta a sus antecedentes. El epifenomenalismo de Huxley, influenciado parcialmente por Descartes, es análogo a un recorrido que tiene dos pasos: (1) la teoría del automatismo animal y (2) los estados conscientes como productos colaterales. Ambos son las dos caras de la misma moneda epifenomenalista ya que sólo si hay una dependencia causal de los estados conscientes podremos hablar de verdaderos "epifenómenos".

\subsection{El primer paso epifenomenalista: la teoría del automatismo consciente}

En Sobre la hipótesis de que los animales son autómatas y su historia, Huxley (1874) lanza interesantes y provocadoras hipótesis sobre la consciencia de los animales, refiriéndose continuamente a la consciencia de los seres humanos puesto que pertenecen al reino animal. En esta teoría, el británico se decanta por un naturalismo epifenomenalista, que, si bien no tiene poder para negar o afirmar nada espiritual, establece el carácter autónomo y determinante del cuerpo en el surgimiento de la consciencia (incluso en la hipótesis de la existencia de un alma espiritual). Para Huxley, los animales son "conscious automata" (Huxley 1874, p. 29), lo cual abre la puerta a concebir al ser humano como un ser natural consciente, sin necesidad alguna de substancias espirituales anejas a su organismo.

En el artículo citado, Huxley se sirve de abundantes citas de Descartes. Pese a que como hemos apreciado se sabe deudor suyo, nuestro científico criticará su postura dualista. Para él, el filósofo francés acertó en su reconocimiento de las leyes autónomas de la naturaleza pero no atinó en absoluto al considerar a los animales -y entre ellos al hombre en sus potencias inferiores- como puras máquinas. La Naturaleza está dotada de una vitalidad propia que llevaría al hombre de a pie -nos advierte Descartes- a confundirla con su dotador supremo, interrogándonos "si Dieu ou la nature auoit formé quelques automates qui imitassent nos actions" (Descartes 1638, p. 41). ${ }^{19}$ Los animales son autómatas que no tienen un contenido cognitivo ni ninguna finalidad consciente en sus acciones. Es muy importante este "si Dios o la naturaleza", expresión que será radicalizada más tarde por el Deus sive natura de Spinoza, así como por un naturalismo que llega a nuestros días; mediante el cual el único punto de vista, el único método y el único objeto de la investigación es lo natural. El naturalista rechaza todo tipo de explicaciones exóticas, o, como mínimo, duda de ellas.

Huxley se siente un gran deudor de la filosofía cartesiana en su proyecto naturalista. Sin embargo, Huxley no está, para nada, de acuerdo con la doctrina cartesiana de las Réponses, para la cual -tal y como nuestro autor parafrasea al galo-:

[E]ven in us, the spirit, or the soul, does not directly move the limbs, but only determine the course of that very subtle liquid which is called the animals spirit, which, running continually from the heart by the brain into the muscles, is the cause of all the movement of our limbs, and often may cause different motions, one as easily as the other" (Huxley 1874, p. 24).

Huxley se opone ferozmente a la tesis dualista de la doble substancia anímica y corpórea: la causa de las mociones espirituales es única (corporal) y es la que da lugar a la consciencia.

No hay pues, en Descartes, una causalidad directa entre el alma y el cuerpo debido a la diferencia esencial de las dos sustancias: la primera es totalmente espiritual y la segunda totalmente material. Lo

19 "[S]i Dios o la naturaleza había formado algunos autómatas que imitasen nuestras acciones". 
que se da es una suerte de "determinación", que Descartes no acaba de explicar adecuadamente. El mismo concepto de "determinación" nos conduce a un campo semántico en el que la heterogeneidad domina las relaciones. Dicha influencia mutua ocurre -según el filósofo- en la glándula pineal, en la cual, de una forma todavía no conocida en su tiempo, tenía lugar la conjunción somático-corporal. Observamos que Descartes -al no aportar razones concluyentes- estaba incubando los principios de un reino natural excardinado del ámbito del espíritu.

Como es posible leer en las Meditaciones acerca de la filosofía primera. Seguidas de objeciones y respuestas (2009), Descartes arranca de una concepción del Cógito - punto arquimédico de su pensamiento- como una realidad de mero pensamiento (una cosa que piensa o res cogitans) que se contrapone a lo material (res extensa). El binomio alma-cuerpo será llanamente decisivo en la filosofía de la mente cartesiana, del cual estamos necesitamos en aras de comprender la mayor originalidad y la mayor falla de esta filosofía. Hubo quienes objetaron al francés de convertir el alma humana en un lánguido espíritu, esto es, en una sombra desprovista de realidad física.

Huxley leerá en las Réponses la creencia de Descartes que hay mociones "which do not depend on the mind at all, such as the beating of the heart, the digestion of food, the nutrition, the respiration of those who sleep [...] and other similar actions, when they are performed without the mind thinking about them" (Huxley 1874, p. 24). En efecto que la tradición medieval, en especial la tomista, distinguía entre el actus hominis y el actus humanus, en el sentido de que el primero era una acción inconsciente del hombre (actos fisiológicos o involuntarios en general); mientras que el segundo constituía aquellas acciones en las que se activaba la libertad y la voluntad humanas siguiendo un fin consciente. Pero esto no significa que la realidad esté partida en dos planos diferentes. Esto quiere no decir, según Huxley, que los animales sean únicamente complejas máquinas. El planteamiento cartesiano supone una instrumentalización del cuerpo -sobre todo en los animales humanos- a causa de que lo concibe cual autónomo artefacto junto a y al servicio de una sustancia superior y más perfecta que es la res cogitans.

El naturalismo de Huxley le lleva a invertir la propuesta de Descartes: su punto de partida no es el Cogito sino el cuerpo. Nuestro científico recurre a ejemplos propios de la neurofisiología y a la experimentación con el fin de fundamentar en mayor grado sus hipótesis. Y es que podemos dejar por sentado que una de las grandes aportaciones del naturalismo -pues no todo en él ha sido tan nefastoes tener a la experiencia observacional como verdadera fuente de reflexión. ¿Qué pasa con la consciencia desde la óptica naturalista? Huxley quiere que notemos que "that it is wholly impossible absolutely to prove the presence or absence of consciousness in anything but one's own brain, though, by analogy, we are justified in assuming its existence in other men" (Huxley 1874, p. 25).

Es misión imposible probar la existencia de la consciencia en otros seres, a excepción de la certeza privada que se origina a partir del funcionamiento del sistema nervioso de cada persona. Poniendo al margen el método introspectivo cartesiano, el británico retoma la analogía aristotélica como medio de tener certeza, si bien no como fruto de la actividad científica, sí como una herramienta del sentido común para asumir que adviene la consciencia en los cerebros de los otros seres. La analogía establece comparaciones a partir de lo conocido por los sentidos y lo imaginado. El éxito, más, estará en dependencia de cómo analicemos observacionalmente el cerebro, el cual -nos dirá sin tapujos Huxley"is the organ of consciousness" (Huxley 1874, p. 25). Nada detectamos aquí de dualismo: la consciencia está ya en la auto-organización biológica. ${ }^{20}$

Nuestro científico fue llamado, con razón, el bulldog de Darwin, ya que llevó hasta las últimas consecuencias - polémicas, las más de las veces- la teoría de la evolución. Según ésta, la constitución de los seres vivos se explica en términos de una tortuosa transformación orgánica, según la ley de una rígida selección (azarosa, han dicho algunos) de los más aptos. En el caso del cerebro, Huxley fue uno de los primeros en defender explícitamente una evolución entre los simios inferiores y el hombre, aunque encuentra severos huecos en la línea evolutiva pues "in the present creation, at any rate, no

\footnotetext{
${ }^{20}$ Otra cosa será -como veremos en el próximo punto- la manera epifenomenalista en que Huxley concibe a la consciencia.
} 
intermediate link bridges over the gap between Homo and Troglodytes" (Huxley 2006, p. 70). Como hemos leído en El puesto del hombre en la Naturaleza (2006), Huxley ya se admiraba de la tremenda carga cualitativa que conlleva el cerebro humano, así como de las estructuras óseas que conforman nuestro esqueleto.

Al compararnos con los simios, el británico consideraba al hombre como perteneciente a una familia aparte. Es maravilloso observar cómo nos separa un abismo entre ellos, aunque Huxley no quiere que perdamos de vista que, si bien sería no menos incorrecto como absurdo negar la existencia de este abismo, "it is at least equally wrong and absurd to exaggerate its magnitude, and, resting on the admitted fact of its existence, to refuse to inquire whether it is wide or narrow" (Huxley 2006, p. 70). Hay otras familias de primates (como la que hay entre el gorila y el orangután, o entre éste y el gibón) cuyas diferencias óseas son mínimas, lo cual no nos concede el libelo para abandonar la empresa naturalista.

Por tanto, "there can be no justification for place him in a distinct order" (2006, p. 70). Huxley entiende la evolución de la mente, en sus estadios progresivos de consciencia, como directamente proporcional a la evolución del cerebro; colocando al ser humano en la milenaria cadena de evolución homínida y, por descontado, animal. Las pruebas paleontológicas hacen más que probable la teoría darwiniana, por lo que así de contundente se muestra el británico sobre el proceso de causación física:

$[T]$ hen it seems to follow that if any process of physical causation can be discovered by which the genera and families of ordinary animals have been produced, that process of causation is amply sufficient to account for the origin of Man (Huxley 2006, p. 71).

Volviendo al artículo de Huxley sobre el automatismo animal, vemos que la consciencia dependerá de cómo se encuentre nuestro sistema nervioso. El británico presenta diversos casos en los que, si el cerebro o la columna vertebral resultasen dañados, también resultaría perjudicada la mente en su actividad consciente. Para nuestro autor el sistema nervioso "is the seat of extremely remarkable powers" (Huxley 1874, p. 25), en fuerte contraste con el carácter tremendamente instrumental y pasivo de lo corporal en Descartes.

Como buen biólogo, el británico puso el ejemplo de una rana, que es sometida a experimentación. Al compararla con los humanos, nos dice que si la rana es capaz de nadar no es por otra cosa que por la impresión causada por el agua en sus nervios sensoriales, la cual produce la transmisión al sistema nervioso de un impulso que origina que sus músculos puedan ser coordinados para nadar (Huxley 1874, p. 26). La ciencia contemporánea, en efecto, da muchas pruebas a favor de que los animales tienen una vida mecánica (muchas más de las que el mismo Descartes poseyó), mas éstas no pueden refutar totalmente que "the frog is not acting from purpose, has no consciousness, and is a mere insensible machine" (Huxley 1874, p. 26). Huxley sentó las bases para hallar rastros de comportamiento consciente en los animales.

En el caso de los seres humanos, Huxley expone un caso estudiado por el afamado Dr. Mesnet de un sargento francés (denominado F) de 20 años que fue herido de bala en el hueso parietal izquierdo del cráneo. Debido a esta grave lesión, al militar le quedó totalmente paralizada, por espacio de un año, la mitad derecha de su cuerpo. Su cuerpo no respondía a ningún estímulo en su lado diestro, ni a pinchazos ni a descargas eléctricas. Lo más remarcable es que el joven atravesaba por momentos de una actividad mental anormal en los que por espacio de unas horas sufría convulsiones o gritos, mientras que cuando regresaba al estado normal de consciencia todo esto desaparecía. Pero, ituvo consciencia el soldado en los momentos de crisis? Huxley responde que "it is impossible to prove that F- is absolutely unconscious in this abnormal state, but it no less impossible to prove the contrary" (Huxley 1874, p. 27).

\subsection{El segundo paso epifenomenalista: la consciencia colateral}

El epifenomenalismo, entendido en sentido tradicional como la corriente que defiende que los estados conscientes mentales, aún siendo productos de la naturaleza física, no pueden afectar causalmente 
dicha naturaleza, concretizada en el cuerpo, debería, por tanto, poder ser explicitado en unas tesis lo suficientemente claras como para detectarlo en Huxley. Según las investigaciones recientes (Walter 2007), tres serían las tesis que compartirían con más generalidad los epifenomenalistas, salvando los matices argumentativos y las adherencias de escuela filosófica de los diferentes pensadores. A la vez que las enunciaremos, buscaremos los razonamientos del británico en donde relucen las siguientes tesis: (a) La tesis de la dependencia causal, (b) la tesis de la irreductibilidad y (c) la tesis de la impotencia causal.

\section{a) Todo evento mental tiene una causa o conjunto de eventos físicos como causa completa (causal dependence thesis)}

El epifenomenalismo de Huxley defiende la relación causal del cuerpo con respecto de los estados conscientes de la mente. Es más, dicha causalidad es completa puesto que no se deben buscar en la filosofía natural causas ajenas a la estructura material de la realidad. De este modo, nuestro científico, enrolado en el método observacional, se refiere a la mente cognoscente en función de la causalidad eficiente: Huxley, como en la tradición filosófica que le precede, quiere llegar a "al principio del que fluye primariamente [la acción consciente] que hace que [la consciencia] sea" (Gay Bochaca 2004, p. 136). Este tipo de causa motriz (causa movens) es lo que intrigaba a Huxley, si se había de cumplir en ella las cualidades clásicas de alteridad, comunicación de la perfección de la causa y preexistencia del efecto.

El británico creyó que el automatismo animal era una hipótesis de Descartes que difícilmente podía ser refutada: aquí estaba siendo consecuente con la tesis de la dependencia causal. El británico rehusaba creer que la consciencia sea algo que adviene repentinamente sin ningún tipo de evolución in natura. "I am not disposed to accept it", nos dice Huxley (1874, p. 28). Pero también en el desarrollo del psiquismo en cada persona: éste se va complejizando con el desarrollo individual. En este aspecto, Huxley es mucho más clásico que el galo pues cumple el viejo adagio medieval según el cual natura non facit saltus, es decir, la naturaleza no hace saltos, sino que más bien deviene y se transforma. Nuestro autor es partidario de lo que él bautiza como "doctrina de la continuidad" con la cual descarta de plano "that any complex natural phenomenon comes into existence suddenly, and wihout being preceed by simpler modifications" (Huxley 1874, p. 28). Si así fuera, veríamos tirada por el suelo la teoría darwinista: un fenómeno tan complejo como la consciencia no puede aparecer por primera vez en el hombre.

A Descartes le faltó - por estar pendiente de reminiscencias metafísicas- comprender la mente no como algo alentado directamente por Dios en el cuerpo, sino a través de un proceso natural que va de lo más simple a lo más complejo. Huxley pone el ejemplo del desarrollo individual: la mente acrecienta sus capacidades conscientes desde el bebé hasta el adulto. Dicho ejemplo debemos extrapolarlo a las especies: hay consciencia en el reino animal, aunque no del mismo modo ni con la misma fuerza. Todo depende del tipo de sistema nervioso que se tenga: "the lower animals possess, though less developed, that part of the brain which we have every reason to believe to be the organ of consciousness in man" (Huxley 1874 , p. 28.). La Naturaleza no es aquella realidad pasiva sino, como es el caso particular del cerebro, verdadera sede de consciencia y dotadora de los demás poderes mentales.

No es que Huxley le quite la razón a Descartes en su tratamiento de los animales como artefactos más o menos sofisticados. Lo que no acepta nuestro autor es el intento cartesiano de privar a la Naturaleza de su poder creativo y de obligarla a mendigar con respecto a entes espirituales o incluso divinos. Ante el dualismo cartesiano, nuestro autor ataca con un monismo naturalista. Para explicar la consciencia no hay que ir fuera o al lado de la Naturaleza: es en ella donde deberemos anclarnos si perseguimos esclarecer la cuestión. En cuanto a los animales, éstos, del modo en que su organismo esté organizado, son en verdad conscientes. Según Huxley los animales poseen ciertos grados de consciencia, muy inferiores al de los hombres:

[T]he brutes, though they may not possess, our intensity of consciousness, and though, from the absence of language, they can have no trains of feelings, yet have a conciousness which, more or less distinctly, foreshadows our own» (Huxley 1874, p. 28). 
Esta última expresión nos parece más esclarecedora: la consciencia animal prefigura la nuestra. Aún más radical seríamos, si entendiéramos la consciencia animal como la nuestra consciencia en sus forma más primitivas, esto es, tal y como la tuvimos milenios atrás. Para Huxley la consciencia está causada, totalmente, por el cambio molecular (entonces la neurología se hallaba en un estado todavía muy poco elaborado) del órgano cerebral. Si bien no de manera mostrenca y sin servirse de este término, el británico pensó que la consciencia, de la forma que fuese, era una suerte de derivado de la Naturaleza. Veamos esquemáticamente en el siguiente gráfico cuál es el proceso fisiológico por el que los animales (y el hombre) atraviesan hasta llegar a la consciencia:

1. SENSACIÓN

2. AFECCIÓN DE LOS NERVIOS SENSORIALES

3. CAMBIOS MOLECULARES EN EL CEREBRO (“IDEAGENOUS MOLECULES”) ${ }^{21}$

4. ESTADOS DE CONSCIENCIA.

En el caso del funcionamiento del cerebro humano, no hay que apreciarlo como causa externa sino como causa directa y gradual a la vez: su actividad es el antecedente más próximo del surgir la consciencia. Los datos brutos pasan por la sensación (1), afectan los nervios sensoriales (2) y, gracias a las sinapsis neuronales (3), llegan a la mente consciente (4). Si negáramos los pasos 1-3, los eventos conscientes no podrían tener lugar, como si fuese un hálito insuflado por un ente extraño o coordinado por él. No es que Huxley abra fuego contra el ocasionalismo de Geunlicx o Malebranche, ni mucho menos con la doctrina de la armonía preestablecida de Leibniz. Estos esquemas de pensamiento, como la idea Dei cartesiana, tenían que recurrir al ente divino para explicar por qué las sustancias de naturaleza contraria actuaban de modo acompasado y regular.

Mas en Huxley sólo hay un tipo de causas para un tipo de substancias: las naturales. Nuestro autor considera que la ciencia no tiene potestad para negar una concomitancia espiritual en las causas de la Naturaleza, aunque es mucho más fructífero trabajar por encontrar esta influencia poderosa en la Naturaleza misma. El británico quiere evitar el peligro de detener la investigación científica por culpa de unos conocimientos prefijados de antemano por creencias aún por contrastar empíricamente. Por ello, es experimentalmente demostrable para Huxley que las mociones del sistema nervioso son las causas totales y no parciales de los estados conscientes.

\section{b) Ningún evento mental es idéntico o reductible a un evento físico (irreducibility thesis)}

Fijémonos en la disyunción de la segunda tesis: el estado mental podría ser "idéntico o reductible" a su causa eficiente completa. Creemos que es positivo señalar esto pues hay autores para los que la consciencia es idéntica "y" reductible a las operaciones cerebrales, como fue el caso de la reducción psiconeural de Patricia Churchland en Neurophilosophy (1986), obra que eliminaba la consciencia como a lo que no tener en cuenta desde el método filosófico tradicional. Esa nueva filosofía de la consciencia no podía traspasar los límites de las neurociencias, sino que solamente tenía que hacer un análisis riguroso, eso sí- de sus resultados. Para Huxley, sin embargo y de una forma que se parece al moderno naturalismo metodológico humilde que más arriba explicábamos, la identidad psiconeural no deja de ser un postulado metafísico que no puede sino mantenerse hipotéticamente, so pena de traicionar a la ciencia natural.

Lo que le preocupaba a Huxley fue, sin embargo, establecer la irreductibilidad de las cogniciones mentales. Sobre los fenómenos de la consciencia, nuestro autor afirmaba en El progreso de la ciencia que "along with certain transformations of energy, cannot be interpolated in the series of these transformations, inasmuch as they are not motions to which the doctrine of the conservation of energy

${ }^{21}$ Según Huxley, éstas son, de un modo extrañamente perceptible, las moléculas de la memoria (Huxley 1874, p. 29). Éstas se encargan de ir almacenando y procesando la información que captamos sensorialmente, siendo clave en el funcionamiento memorístico del cerebro. 
applies" (Huxley 1887, p. 95). Los estados de la consciencia no pueden ser explicados reductivamente como la serie de transformaciones materiales que los causan debido a su naturaleza diversa: no pueden ser estudiados desde la estricta teoría de la conservación y transformación de la materia energética.

Nuestro científico, pues, reconocía que es experimentalmente cierto que una particular moción molecular hace brotar un estado consciente pero "the how and why of the process are just as inexplicable as in the case of the communication of kinetic energy by impact" (Huxley 1887, p. 95). El cómo y el porqué de los fenómenos mentales no pueden esclarecerse mediante la vía experimental en el momento en que se encontraba la neurología de su época. De este modo, la irreductibilidad inexplicable de los estados de consciencia, considerados en sí mismos, es un rasgo típicamente epifenomenalista en Huxley. Nuestro científico se admiraba ante los importantes descubrimientos de la estática y la dinámica de su tiempo, al poder deducir los fenómenos exhibidos por los cuerpos materiales "from physico-mathematical first principles" (Huxley 1887, p. 96), si bien se plañía que las cogniciones eran indeductibles de nada al acopio de la investigación de su tiempo.

En su trabajo Sobre las bases físicas de la vida (1868), elaboración de una conferencia dictada en Edimburgo, Huxley postulaba el protoplasma como la estructura más profunda de la vida, en un momento en que la biología apenas empezaba a distinguir entre el citoplasma y el núcleo de la célula. Se pensaba, en ese momento, que la célula misma era el componente más simple de la vida, aun estando lejos de la biología molecular y de sus logros en genética. A propósito de la relación entre la materia, el espíritu y los estados conscientes, nuestro científico se preguntaba lo siguiente:

For, after all, what do we know of this terrible "matter", except as a name for the unknown and hypothetical cause of states of our own consciousness? And what do we know of that "spirit" over whose threatened extinction by matter a great lamentation is arising, like that which was heard at the death of Pan, except that it is also a name for an unknown and hypothetical cause, or condition, of states of consciousness? In other words, matter and spirit are but names for the imaginary substrata of groups of natural phenomena (Huxley 1868, p. 161).

Los fenómenos mentales no pueden evadirse mediante el recurso a los nombres "materia" o "espíritu". Según Huxley, estos no serían sino los nombres apresurados de las desconocidas e hipotéticas causas de nuestros estados conscientes. Así mismo, se acerca al nominalismo ockhamista y a la filosofía de David Hume cuando lamenta que se llame "espíritu" a lo que pudiera ser, no una causa, sino uno conjunto de causas desconocidas empíricamente. La falacia causa pro causa es negada por nuestro científico pues lo que sucede antes que otra en la simple aprehensión no constituye per se la causa de sus efectos. El materialismo, para el que no hay nada en el mundo salvo materia, fuerza y necesidad, "is as utterly devoid of justification as the most baseless of theological dogmas" (Huxley 1868, p. 162). Igual tarea de fundamentación necesita el que piensa que todo se reduce a materia como los que acometen la labor reductiva hacia el espíritu. Ni el cómo ni el porqué se justifican racionalmente en el materialismo o en el espiritualismo sino que simplemente enuncian unas tesis de partida, dándolas por probadas. Huxley deniega, de este modo, cualquier base científica a los monismos metafísicos.

El británico tiene una convicción personal, no provenida de la investigación científica, de que algún día se llegará por su medio a demostrar dicha identidad. Huxley admitió que tanto el materialismo como el ateísmo, en sus versiones más fuertes, superan con creces las fronteras de la investigación: "the fundamental doctrines of materialism, like those of spiritualism, and most other 'isms', lie outside 'the limits of philosophical inquiry'” (Huxley 2009a, p. 158). Lo que cabe, como la religión de Kant, es la esperanza, especialmente en que algún día la ciencia natural pueda resolver el problema de la consciencia. Así las cosas, la metodología científica que privilegia el británico le lleva a situarse, tan sólo conjeturalmente, más del lado materialista: en sus Sermones laicos, Huxley no desespera en la convicción "that we shall, sooner or later, arrive at a mechanical equivalent of consciousness" (Huxley 2009a, p. 372). Es, finalmente y aunque sin abandonar su precomprensión naturalista, una postura mucho más modesta y, además, muchos más consecuente con asuntos tan limítrofes. 


\section{c) Ningún evento mental es causa, ni parcial ni completa, de ningún otro evento (causal impotence thesis)}

La tesis de la impotencia causal fue manifiestamente patrocinada por Huxley, la cual es directamente proporcional a la tesis de la dependencia causal: según el británico "all states of consciousness in us [como en los animales] are immediately caused by molecular changes of the brain-substance" (Huxley 1874, p. 30). No tenía razón James Ward en 1909, cuando en su artículo para la Enciclopedia Británica señalaba que en Huxley "physical changes are held to be independent of psychical" (Robinson 2011). Es principalmente esto lo que Huxley echaba en cara a Descartes y lo que, a nuestros ojos, constituye lo más raro de su epifenomenalismo. ¿Cómo es posible -nos preguntamos- que amparando tan gravemente la proveniencia física de la consciencia, haga de ésta un producto anómalo?

Huxley pone dos ejemplos: el del reloj y el de la locomotora. En el primero, la mente sería como la campana de un reloj que está en marcha en cuanto que cuerpo suyo y la conciencia sería como la respuesta al sonido de la campana. Sin el funcionamiento del reloj (el cuerpo), la campana no sonaría (mente) ni habría ninguna respuesta (consciencia). En el segundo, la consciencia sería semejante al bizarro pitido que genera una locomotora en marcha. Son metáforas que a Huxley le vienen de maravilla para tan siquiera asomarse a superar el dualismo cartesiano. Nuestro científico entiende que los eventos de consciencia son estados no-funcionales o subproductos (by-products), que no forman parte de la composición orgánica cerebral. El estado consciente mental es denominado por Huxley como un "collateral product" (Huxley 1874, p. 29) del mecanismo del cuerpo, es decir, un flujo de consciencia (stream of consciousness) del que no se puede tener evidencia que sea totalmente igual a la excitación sensorial u a otros cambios físicos.

Pese a todo ese producto no deja de ser tal, es decir, un concomitante (concomitant) del funcionamiento molecular del cerebro que forma parte de las series causales involucradas en su producción. Nos hallamos, pues, ante un epifenómeno, un fenómeno causado materialmente pero que, a la hora de querer objetivarlo, se nos vela a la empireia científica. La cualidad epifenomenal es secundaria, acompañando los fenómenos físicos pero en tanto que portadora de una irreductible e ineficaz distinción. La sombra del dualismo de Descartes pesa gravemente en el británico ya que, si la consciencia no es exactamente igual al trabajo neuronal, nos encontramos con un tipo de entidad irreducible, paralela y cuyo ejercicio se realiza en un nivel distinto al cerebral. Huxley se ve pobre metodológicamente para describir exactamente de qué está compuesto dicho producto colateral y para descubrir cómo es, paso a paso, su funcionamiento entitativo. El británico rehúsa realizar una ontología de la consciencia en la que reluzcan las razones de fundamentación de la misma.

Fiel a la empireia, Huxley sólo tiene argumentos para establecer las causas y los efectos de la consciencia, si bien, como señalamos, no el qué de la misma. La consciencia es como el pitido que acompaña a una locomotora, yendo al unísono con su funcionamiento pero estando "completely without any power of modifying that working" (Huxley 1874, p. 29). Aquí detectamos claramente la tesis de la impotencia causal, uno de los elementos más propios de epifenomenalismo: los estados mentales no tienen repercusión alguna en el ámbito natural, si bien están paradójicamente originados en él. Nuestro autor espeta que no se ha comprobado que los estados de consciencia tengan cualquier clase de causalidad en la moción orgánica del cuerpo:

It seems to me that in men, as in brutes, there is no proof that any state of consciousness is the cause of change in the motion of the matter of the organism. If these positions are well based, it follows that our mental conditions are the symbols in consciousness of the changes which takes place automatically in the organism (Huxley 1874, p. 30).

Podríamos, entonces, calificar de exponencial que nuestro autor considere los estados conscientes como símbolos, sin otorgarles la misma entidad que el organismo. La volición guarda este carácter simbólico porque no es la causa de ningún acto voluntario en el comportamiento, sino el símbolo de un estado en el cerebro que es la causa inmediata del acto voluntario. En Huxley, por ello, se trata de símbolos de lo fisiológico mismo. De esta forma, Huxley se muestra partidario de un organismo 
completamente natural que es capaz de dar de sí productos simbólicos que escapan a su autoorganización pero que de facto emergen de la misma y gozan de un tipo -indeterminado- de realidad. Nuestro científico no se atreve a decir que esto es algo sólo espiritual o sólo material, ni mucho menos ambas cosas, debido a la metodología naturalista modesta, que guía sus hallazgos científicos. De hecho, se defiende ante la acusación de herejía al saberse falto de poder justificativo para hacer tales reducciones monistas o separaciones dualistas.

Huxley, pues, inauguró los dos reclamos (Campbell 1997, p. 41) epifenomenalistas "fuertes":

1. Los eventos mentales no tienen eficacia causal.

2. Identificar los estados mentales de un agente nos ofrece una explicación causal de la acción del agente.

Cuando Huxley se refiere a la volición humana como un símbolo del comportamiento autómata del ser humano, de nada le sirve para justificar fehacientemente sus acciones. El primer reclamo se denomina "fuerte" pues da por hecha la impotencia causal, mientras que el segundo también lo es porque se cree avalado, en base al análisis sesgado de los estados conscientes, para concluir que la consciencia no tiene ninguna influencia causal en el ámbito físico. Si probásemos de sustituir el reclamo 2 por una versión débil ("Identificar los estados mentales de un agente no nos ofrece una explicación causal de la acción del agente”) reconoceríamos que no se sigue la resolución del problema de la impotencia causal a partir de la simple identificación y análisis de las acciones conscientes en un individuo. Así pues, el primer punto puede llevarnos al segundo, si bien el débil no puede sostener el planteamiento fuerte, en contra de Huxley. Ciertamente identificar que un agente tenga este o aquel estado mental no es suficiente para despejar la incógnita de su causalidad ya que no es ésta el objeto estudiado, aunque tampoco queda zanjada la cuestión de si en verdad los estados conscientes causaron o no la acción del agente.

Lo expuesto hasta el momento nos facultaría para llamar a Huxley "monista anómalo", a diferencia del monismo neutral de James o Russell. Recordemos que estos filósofos, negando el dualismo epistemológico y ontológico, defendían que el universo consiste únicamente en elementos "neutrales", que no son en sí mismos ni mentales ni físicos. La consciencia no posee ninguna entidad y únicamente en su acceso posterior a las propiedades de los objetos reales se puede hablar de la división, in mente, de lo físico o lo mental. Así por ejemplo, el color o la forma de las cosas, en tanto que experiencias conscientes, no existen en la mente al modo epifenomenal ni tampoco únicamente como producto neurológico, sino que existen en sí mismas en las cosas en tanto que propiedades neutrales que son clasificadas y aprehendidas realísticamente por la mente. Huxley tacharía a los monistas neutrales de preservar un postulado dogmático debido a que no tenemos a nuestro alcance las pruebas empíricas para identificarla sin más con las mociones corporales.

Huxley aboga, por el contrario, por la existencia de la consciencia y el carácter cualitativamente distinto de sus estados, si bien mantiene un monismo naturalista en su metodología. El británico tiene bien clara la proveniencia natural de la consciencia, si bien recula ante la posibilidad de investigar y describir estrictamente en qué consiste. La anomalía de su monismo comparece cuando epifenomenaliza la consciencia en cuanto que irreductible y colateral; y todavía más cuando postula, sin demasiadas razones a su favor, que no modifica al organismo. Donald Davidson, en Mental Events (1970), fue partidario una forma semejante de monismo anómalo. Como Huxley, cada evento mental depende causalmente de un evento físico pero, aunque Davidson diría que son la misma realidad, no contamos con leyes psicofísicas para los eventos mentales. Es decir, lo psíquico y lo neural no son exactamente la misma cosa, según Huxley. Todo evento mental cuenta con un evento neurofisiológico en que se apoya y la descripción del mismo -pensó Davidson- no nos vale para predecir los pensamientos y acciones voluntarias del agente intencional. Otra discrepancia con nuestro científico es que Davidson optará por una teoría fisicalista de la identidad mente-cerebro, negando la tesis huxleyana de la irreductibilidad. Lo que tienen en común, sin embargo, es el rasgo anómalo de los eventos mentales. 
Pese a todo, esto no nos puede conducir a un epifenomenalismo de manera forzada, habida cuenta de la réplica explicativa de Campbell (2005), por la que la identificación de las propiedades mentales de A no aporta la explicación causal del comportamiento de A. No todo monismo anómalo ha de ser necesariamente epifenomenalista ya que no tener leyes físicas para la consciencia no significa de por sí que no tenga efectos físicos. Huxley excede, debido a lo que explicábamos más arriba, este tipo de epifenomenalismo explicativo débil: "Identificar los estados mentales de un agente no nos ofrece una explicación causal de la acción del agente". Nuestro científico defendió lo contrario, también excediendo la configuración de su naturalismo modesto.

\section{Conclusiones}

Terminemos nuestro artículo con unos breves y esquemáticos puntos en donde salgan a relucir, resumidamente, las principales conclusiones a la que nos ha llevado el análisis que hemos acometido del pensamiento de Huxley y de Descartes. No podemos sino afirmar el carácter abierto, tanto para el derecho a réplica como al de puntualización, que cualquier investigador quiera ejercer:

1) Huxley hizo de la naturaleza el ámbito totalizante de la vida, con tremendas consecuencias en la ética. Independientemente de que exista un más allá, la naturaleza es todo y lo único que se da a nuestra experimentación. Cada vez que Huxley usa el concepto "natural" se refiere solamente a lo que es material. No es menos llamativo que Huxley intuya una moral iusnaturalista que no llega a desarrollar filosóficamente. El naturalismo de Huxley es diametralmente opuesto al ontológico puesto que la tesis por la que todo es materia la formula sólo como una hipótesis aún por verificar científicamente y no como presupuesto o primer principio.

2) Según nuestro autor, la ciencia empírica, cuyo método es la observación, pretende hacer demostraciones que tengan el rigor indispensable de la evidencia. Esta ciencia, para Huxley, es reveladora de la verdad pero como algo a conquistar y no como algo ya conquistado: el británico, influenciado por Hume, declaró la guerra al espiritualismo y al materialismo por encontrarlos "dogmáticos".

3) Huxley conoció de primera mano los textos de Descartes y se atrevió a pensarlos por sí mismos, pese a la archiconocida acusación de racionalismo que pesaba sobre el francés. Nuestro autor, pues, no hizo de la ciencia empírica un gueto, que quisiese refugiarse de todo lo que sonase a humanidades, como sucedió en la primera mitad del siglo XX con el cientificismo. Así pues, Huxley fue, de forma impresionante, un autodidacta que se preocupó por hacer que la ciencia tuviese que ver con la totalidad de la vida del hombre.

4) Huxley fue cartesiano, al menos, en lo que al enfoque naturalista se refiere. Nuestro científico encontró en Descartes las rompedoras afirmaciones de una naturaleza material que gozaba de autonomía y dinamicidad propias. Un ejemplo de ello es que, tanto Huxley como el galo, tienden a personificarla informalmente cual artífice viviente y creadora de actos conscientes. Por el contrario, Huxley se opone a la teoría de la doble substancia: sólo hay una substancia (la material) y sólo de ella proceden los eventos mentales. Podemos llamar sin rubores a Huxley "naturalista" pero es un "naturalista humilde o modesto" (Giere 2007), al ir de la mano con los descubrimientos científicos a la hora de hacer afirmaciones concluyentes. En esta guisa, Huxley no concibe su caracterización como unilateralmente excluyente de la existencia de dioses o almas puesto que, rechazarlos o no, depende en gran parte de una ciencia que se encuentra en un estadio aún bastante pobre. La postura de Huxley en relación al misterio de Dios es la del agnosticismo.

5) Huxley, por el contrario y del mismo modo que ve a la Naturaleza como dadora de vida, entiende el cuerpo natural de modo activo en la consciencia humana, en calidad de epicentro de potencialidades cognitivas. El británico sostiene la tesis de que el cuerpo (animal u humano), mediante el organismo cerebral, es sede de consciencia y no un burdo 
utensilio anejo a una sustancia espiritual. El mismo dato de llamar al hombre un autómata consciente muestra su interés en superar la antropología dualista del galo. Si la postura cartesiana destaca por su extrincesismo, la de Huxley lo hace por su intrinsecismo. Descartes, como hemos apreciado, recurre a Dios como aquella idea idearum, sin la cual no sería concebible ni posible nada, en especial la naturaleza. Huxley abre, por el contrario, caminos para considerar más positivamente a la naturaleza, incluso como causa por la que y en la que la consciencia puede emerger y desplegarse.

6) Si Descartes acaba en un pensamiento dualista, Huxley desemboca en un monismo anómalo, de una sola res que da lugar a una res mental consciente que no se rige exactamente por las leyes psicofísicas de la primera. El británico fue uno de los mayores abanderados del epifenomenalismo, gracias a su propuesta de la emergencia natural de la consciencia, de un modo inmediato en cuanto producto emergente colateral del cerebro y cuya entidad inexplicable acaba por detener la secuencia causal originante (no puede alterar lo natural de lo que procede). Huxley, pues, entiende los estados de la mente consciente como símbolos de la naturaleza misma, razón por la que echamos de menos una descripción más detenida y justificada en el británico de este asunto si no quiere ser calificado, como el galo, de dualista.

En vez de las contemporáneas neuronas, son concomitantes a estos símbolos unas enigmáticas moléculas por las que el cerebro procesa la información que proviene de los sentidos, lo cual se explica en un momento en que la neurología apenas estaba siendo inaugurada. De este modo, los estados conscientes dependen causalmente del funcionamiento cerebral, siendo irreductibles al mismo y -ésta es la anomalía- siendo impotentes causalmente de cara al milieu físico originante. Huxley, al contrario que el fisicalismo epifenomenalista de Davidson (1970), no identifica los estados mentales con los cerebrales sino que paraleliza su cualidad. Así mismo y a diferencia del epifenomenalismo débil (Campbell 2005), se puede decir, finalmente, que Huxley parece ser un monista en el origen pero un dualista en la meta, sobre todo por su silencio sobre qué tipo de cualidad tienen los eventos de la consciencia. Pese a esto, Huxley creía que los estados conscientes de un agente nos ofrecen una explicación causal (ineficaz, nos ha dicho) de la acción del agente, cosa que los críticos actuales consideran innecesaria y precipitada en el británico.

Bibliografía

Caston, V. (1997), "Epiphenomenalisms, Ancient and Modern", The Philosophical Review 106: 309-363.

Campbell, N. (1997), Physicalism and the Challenge of Epiphenomenal Properties, McMaster University, Tesis doctoral, https://macsphere.mcmaster.ca/handle/11375/15600.

Campbell, N. (2005), “Explanatory Epiphenomenalism”, The Philosophical Quarterly 55: 437-451.

Churchland, P.S. (1986), Neurophilosophy: Toward a Unified Science of the Mind-Brain, Cambridge, MA: MIT Press.

Davidson, D. (1970), "Mental Events", en Foster, L. y J.W. Swanson (eds.), Experience and Theory, London: Duckworth, pp. 79-101.

Descartes, R. (1637), Discours de la méthode $\mathcal{G}$ essais, en Adams, C. y P. Tannery (eds.), Oeuures complètes, vol. VI, París: Cerf, 1902.

Descartes, R. (1638), “Lettre CXIII”, en Adams, C. y P. Tannery (eds.), Oeuvres complètes, vol. VI, París: Cerf, 1898.

Descartes, R. (1639), "Lettre CLVII à Mersenne”, en Adams, C. y P. Tannery (eds.), Oeuures complètes, vol. VI, París: Cerf, 1898.

Descartes, R. (1959), Dos opúsculos. Reglas para la dirección del espiritu. Investigación de la verdad, México: UNAM. 
Descartes, R. (2009), Meditaciones acerca de la filosofía primera. Seguidas de objeciones y respuestas, Bogotá: Universidad Nacional de Colombia.

Gay Bochaca, J. (2004), Curso de filosofía, Madrid: Rialp.

Giere, R.N. (2007), “Modest Evolutionary Naturalism”, en Mi, C.M. y R. Chen, (eds.), Naturalized Epistemology and Philosophy of Science, Amsterdam: Rodopi, pp. 21-37.

Hodgson, S.H. (1970), The Theory of Practice, Londres: Longas, Green, Reader and Dyer.

Huxley, T.H. (1868), "On the Physical Basis of Life”, en Huxley, T.H., Methods and Results. Essays, Nueva York: D. Appleton and Co., 1898, pp. 130-165.

Huxley, T.H. (1874), "On the Hypothesis that Animals are Automata, and its History", en Chalmers, D.J. (ed.), Philosophy of Mind. Classical and Contemporary Readings, Nueva York: Oxford University Press, 2002, pp. 24-30.

Huxley, T.H. (1887), “The Progress of Science”, en Huxley, T.H., Methods and Results. Essays, Nueva York: D. Appleton and Co., 1898, pp. 42-129.

Huxley, T.H. (2006), Man's Place in Nature. The Evolution Debate: 1813-1870, Vol. II, London: Routledge,

Huxley, T.H. (2009a), Lay Sermons, Adresses and Reviews, Cambridge: Cambridge University Press.

Huxley, T.H. (2009b), Essays upon some Controversed Questions, Cambridge: Cambridge University Press.

Huxley, T.H. (2009c), Evolution and Ethics. Delivered in the Sheldonian Theatre, May 18, 1893, Cambridge: Cambridge University Press.

James, W. (2007), The Principles of Psychology, Nueva York: Cosimo.

Mackie, J. (1990), Ethics: Inventing Right and Wrong, London: Penguin Books.

Papineau, D. (2007), "Naturalism”, en Zalta, E.N. (ed.), The Stanford Enclyclopedia of Philosophy, URL = http://plato.stanford.edu/cgi-bin/encyclopedia/archinfo.cgi?entry=naturalism (acceso 1 de enero, 2014).

Moore, G.E. (1913), Principia Ethica, Londres: Cambridge University Press.

Quine, W.V. (1992), Pursuit of Truth, Cambridge, MA: Harvard University Press.

Robinson, S. (2012), en Zalta, E.N. (ed.), The Stanford Enclyclopedia of Philosophy, URL = http://plato.stanford.edu/cgibin/encyclopedia/archinfo.cgi?entry=epiphenomenalism (acceso 1 de enero, 2014).

Rus, S. (1994), "La falacia naturalista en la Grecia Clásica”, Persona y Derecho 30: 321-343.

Walter, S. (2007), "Epiphenomenalism”, http://www.philosophy-online.de/pdf/ohpm.pdf (acceso 1 de enero, 2014).

White, P. (2003), Thomas Huxley. Making the "Man of Science", Cambridge: Cambridge University Press. 